\title{
Interstitial Brachytherapy in Combination With Previous Transarterial Embolization in Patients With Unresectable Hepatocellular Carcinoma
}

\author{
DIRK SCHNAPAUFF ${ }^{1 *}$, BRUNO R. TEGEL $^{1 *}$, MACIEJ J. POWERSKI ${ }^{2}$, \\ FEDERICO COLLETINI ${ }^{1}$, BERND HAMM ${ }^{1}$ and BERNHARD GEBAUER ${ }^{1}$ \\ ${ }^{1}$ Department of Radiology, Charité Universitätsmedizin Berlin, Campus Virchow-Klinikum, Berlin, Germany; \\ ${ }^{2}$ Department of Radiology and Nuclear Medicine, Otto-von-Guericke University, Magdeburg, Germany
}

\begin{abstract}
Background/Aim: Treatment of patients with large hepatocellular carcinoma $(\mathrm{HCC})$ remains challenging and survival in advanced tumor stages is limited. This study was conducted to investigate the efficacy of embolization followed by computed tomography (CT)-guided interstitial high-dose-rate brachytherapy (CT-HDRBT) in patients with unresectable HCC. Patients and Methods: A total of 47 patients undergoing CT-HDRBT were divided into 2 groups: i) patients previously treated with transarterial chemoembolization (TACE) and ii) patients treated with bland transarterial embolization (TAE). The primary endpoint was overall survival (OS), while secondary endpoints were the time to progression (TTP) and the local progression rate. Results: A total of 78 lesions were treated. The mean size of the main tumors was $58.3 \mathrm{~mm}$. The median OS in TACE and TAE groups was 28.9 months and 32.3 months, respectively $(p=N S)$. The median $O S$ of patients classified as BCLC stage A using the Barcelona Clinic Liver Cancer classification system (BCLC) was 32.3 months, while the median $O S$ of patients in BCLC stage $B$ and $C$ was 36.9 and 17.7 months, respectively. The local progression rate was $7.7 \%$ (6/78), with no statistically significant difference between TACE and TAE. The median TTP was significantly longer in the TACE group compared to the TAE group (11.7 months and 10.3 months, respectively). Conclusion: Treatment with transarterial embolization and subsequent
\end{abstract}

\footnotetext{
*These Authors contributed equally to this work.

Correspondence to: Dirk Schnapauff, Charité Universitätsmedizin Berlin, Campus Virchow-Klinikum, Department of Radiology, Augustenburger Platz 1, 13353 Berlin, Germany. Tel: +49 30450657267, Fax: +49 304507657267, e-mail: dirk.schnapauff@ charite.de
}

Key Words: Hepatocellular carcinoma, embolization, brachytherapy, radiosensitizing effect, survival, interventional radiology.
CT-HDRBT leads to a very promising survival rate for patients with unresectable HCC.

Endovascular and percutaneous interventions play an important role in the treatment of patients with hepatocellular carcinoma (HCC), as descripted in clinical management guidelines based on the Barcelona Clinic Liver Cancer (BCLC) staging system (1). According to the BCLC system, surgical approaches, such as liver resection and liver transplantation, as well as imageguided tumor ablation are curative treatments for very early (BCLC 0) and early stage tumors (BCLC A) (1).

Patients with an intermediate stage (BCLC B) HCC are candidates for trans-arterial chemoembolization (TACE), as randomized trials have proven the efficacy of this technical approach to control symptoms and prolong survival (2).

However, TACE remains a palliative treatment with an unsatisfactory survival of 16-16.9 months in patients with BCLC stage B and 6-10.7 months in patients with BCLC stage $\mathrm{C}(1,3)$.

In recent years, the combination of transarterial embolization (TAE) and percutaneous ablation, such as radiofrequency ablation (RFA), has been established and is widely applied (4). Using this combination, complete thermal ablation can be achieved in HCCs that are up to $5 \mathrm{~cm}$ in diameter, whereas thermal ablation alone leads to high rates of incomplete ablation and early local recurrence in HCCs larger than $3 \mathrm{~cm}(5,6)$.

To overcome the size limitation of thermal ablation techniques, CT-guided interstitial high-dose rate brachytherapy (CT-HDRBT) that uses high dose gamma radiation was developed for tumor ablation (7). In addition, since radiation is not subjected to the heat sink effect, CTHDRBT is also applicable for lesions that are in direct contact to major vessels (8).

Cisplatin is one chemotherapeutic agents used in TACE, and is known to have a radiosensitizing effect on tissue, leading to higher vulnerability to gamma radiation $(3,9)$.

The aim of this study was to investigate the survival of patients with an intermediate- to advanced-stage HCC who 
have been treated with transarterial embolization and subsequently by CT-HDRBT.

\section{Patients and Methods}

All procedures performed in this study that involve human participants were in accordance with the ethical standards of the institutional and/or national research committee and with the 1964 Helsinki declaration and its later amendments or comparable ethical standards (10). The study was approved by the Institutional Review Board (No. EA2/114/10). Informed consent was obtained from all individual participants included in the study. For this type of study consent for publication is not required.

Patients. Baseline characteristics of the cohort are summarized in Table I.

We included 47 Patients that presented with unresectable HCC between March 2011 and March 2015. Diagnosis was confirmed by imaging criteria and in most cases (35/47) by additional histological assessment. All patients were discussed in the multidisciplinary tumor board and their inclusion in the study was decided by a consensus.

All patients underwent a dedicated MRI of the liver with a liver specific contrast medium (EOB-DTPA, Primovist, Bayer Pharma, Leverkusen, Germany) and a multislice contrast-enhanced spiral CT of the chest, abdomen and pelvis, to rule out extrahepatic tumor burden or secondary malignancy.

Following baseline imaging, patients were treated with TACE and subsequent CT-HDRBT. As transarterial embolization with Lipiodol makes tumors clearly visible in native CT scans, a second group of patients who underwent CT-HDRBT following bland TAE was collected to rule out any bias due to a facilitated catheter placement in CT fluoroscopy. Data collection and analysis were performed under the approval of the ethical review board, Number EA2/114/10.

Embolization. All transarterial procedures were carried out under sterile conditions using latest-generation digital substraction units, under local anesthesia and when necessary with mild conscious sedation, using 2-3 mg Midazolam (Hoffmann-La Roche, Basel, Switzerland). In the TACE arm, embolization was performed with an emulsion of $10 \mathrm{ml}$ iodised oil (Lipiodol, Guerbet, Aulnay-sousBois, France) and $50 \mathrm{mg}$ cisplatin (Medac, Wedel, Germany) diluted in $5 \mathrm{ml}$ saline. As crystalline cisplatin was withdrawn from the market in 2013, it was replaced by $50 \mathrm{mg}$ mitomycin C (Medac, Wedel, Germany), which created a second TACE group (mitomycinTACE). In the bland embolization arm, embolization was performed with iodized oil only.

CT-guided interstitial high dose rated brachytherapy. One day following embolization all patients underwent CT-HDRBT. Treatment was performed using a conscious sedation. Working steps of CT-HDRBT have been explained in detail in several publications $(7,8,11)$. Briefly, following a spiral CT scan of the upper abdomen the expected puncture sites were marked and sterilized. Subsequently, tumors were punctured using a $17 \mathrm{G}$ needle and 6 French $(6 \mathrm{~F})$ angiographic sheaths were pushed into the tumor over a stiff guide wire. These sheaths served as stabilizing devices for the closed ended $6 \mathrm{~F}$ catheters, which were introduced subsequently. Catheter positions in relation to the tumor were depicted in the native CT scan, which was used for further radiation planning using a three-dimensional radiation planning workstation (Brachyvision, Varian Medical Systems, Palo Alto, CA, USA). The intended dose applied to the clinical target volume (CTV) was 20 Gy with a sharp dose descent, sparing the tissue outside the tumor margin. Radiation was performed in a single fraction using an Iridium 192 source. Finally, catheters were retracted, and the puncture tracts were sealed using thrombogenic sponge torpedoes to minimize the risk of bleeding.

Follow-up. Patients were followed-up by regular clinical visits and MRI of the liver, starting at 6 and 12 weeks following CT-HDRBT, and then spacing them by 3 months. Eighteen months later the intervals between MRI scans were extended to 6 months. When local recurrence (defined as asymmetric lesion growth at any time during follow-up) or new tumors at distant sites occurred, a second or third therapy was applied in cases treatable by CT-HDRBT in combination with TACE or TAE (according to the group patients were assigned to).

The follow-up ended in April 2018, and survival was determined either by personal interview or from data obtained from the residents' registration office or the local cancer registry.

Endpoints and statistical analysis. The primary endpoint was Overall Survival (OS) and the secondary endpoints were: i) Time to Progression (TTP) and ii) local progression rate.

Baseline characteristics were reported using descriptive statistics, and differences between the treatment groups were calculated using multivariate analysis in a cox regression model. OS and TTP were calculated using the Kaplan Meier Method with the SPSS Software Package Version 25 (SPSS, IBM, Armonk, NY, USA). Statistical significance was defined by a $p$-value of less than 0.05 in the Log-rank test. Adverse events were reported according to the Society of Interventional Radiology (SIR) criteria (12).

\section{Results}

Study cohort and intervention. Forty-seven patients with 78 tumors were enrolled in the study. The mean age was 70.7 years in the TACE group and 70.8 in the TAE group. The male/female ratio was $5: 1$ in both groups. The mean diameter of the main lesion was $58.3 \mathrm{~mm}$ in the TAE group, $47.5 \mathrm{~mm}$ in the cisplatin-TACE group and $57.1 \mathrm{~mm}$ in the mitomycin-TACE group. In total, 4 patients were BCLC stage A, 30 patients were BCLC stage B and 13 patients were BCLC stage C. All BCLC stage $C$ cases involved segmental portal venous invasion.

Patients' baseline characteristics are listed in detail in Table I, the 2 TACE arms are listed separately with respect to the drug used and are summarized as c-TACE groups.

Procedural outcome. A total of 19 patients underwent bland TAE followed by CT-HDRBT (Figure 1). Twelve patients were treated with cisplatin-TACE (Figure 2) and subsequently by CT-HDRBT, while 16 patients were treated with mitomycin-TACE followed by CT-HDRBT. According to the 
Table I. Baseline characteristics of the study cohort according to treatment modality. cTACE patients are further divided by drug used. $p$-Value indicates whether there were significant differences between the groups.

\begin{tabular}{|c|c|c|c|c|c|}
\hline & $\begin{array}{c}\text { TAE + } \\
\text { Brachytherapy }\end{array}$ & $\begin{array}{c}\text { cTACE + } \\
\text { Brachytherapy }\end{array}$ & $p$-Value & $\begin{array}{l}\text { cTACE- } \\
\text { Cisplatin }\end{array}$ & $\begin{array}{c}\text { cTACE- } \\
\text { Doxorubicin }\end{array}$ \\
\hline Number of patients & 19 & 28 & & 12 & 16 \\
\hline Mean age, years (SD) & $70.8(6.8)$ & $70.7(11.5)$ & 0.601 & $66.3(11.6)$ & $72.2(12.2)$ \\
\hline Gender (male/female) & $16 / 3$ & $23 / 5$ & 1 & "11/1" & "23/5" \\
\hline \multicolumn{6}{|l|}{ Lesion } \\
\hline Multifocal HCC & $6(31.6)$ & $16(57.1)$ & 0.085 & $7(58.3)$ & $9(56.3)$ \\
\hline Mean number of lesions (range) & $1.32(1-2)$ & $2.03(1-5)$ & 0.225 & $2.17(1-5)$ & $1.94(1-5)$ \\
\hline Mean diameter main lesion (range) & $58.32(23-144)$ & $55.74(22-110)$ & 0.443 & $47.5(22-86)$ & $57.12(30-110)$ \\
\hline Portal invasion (\%) & $4(21.1)$ & $8(28.6)$ & 0.737 & $3(25)$ & $5(31.3)$ \\
\hline BCLC-Stage (\%) & & & 0.342 & & \\
\hline A & $3(15.8)$ & $1(3.6)$ & & $1(8.3)$ & 0 \\
\hline $\mathrm{B}$ & $12(63.2)$ & $18(64.3)$ & & $8(66.7)$ & $9(56.3)$ \\
\hline $\mathrm{C}$ & $4(21.1)$ & $9(32.1)$ & & $3(25)$ & $7(43.8)$ \\
\hline \multicolumn{6}{|l|}{ Liver function } \\
\hline Ascites (\%) & $2(10.5)$ & $5(17.9)$ & 0.685 & $1(8.3)$ & $4(25.0)$ \\
\hline Hepatic encephalopathy (\%) & $1(5.3)$ & $1(3.6)$ & 1 & 0 & $1(6.3)$ \\
\hline Cirrhosis $(\%)$ & $17(89.5)$ & $27(96.4)$ & 0.557 & $11(91.7)$ & $16(100)$ \\
\hline Alcohol-abuse (\%) & $9(50)$ & $10(41.7)$ & 0.591 & $4(33.3)$ & $6(37.5)$ \\
\hline Hepatits B (\%) & 0 & 0 & & 0 & 0 \\
\hline Hepatitis C (\%) & $3(15.8)$ & $8(38.1)$ & 0.163 & $6(50)$ & $1(6.3)$ \\
\hline Mean AFP, $\mu \mathrm{g} / 1$ (SD) & $706.8(2506.7)$ & $21485.8(102057.8)$ & 0.624 & $32.36(11.57)$ & $37219.15(34614.77)$ \\
\hline Mean albumin, g/dl (SD) & $5.0(4.89)$ & $3.7(0.704)$ & 0.263 & $3.73(0.75)$ & $3.63(0.69)$ \\
\hline Mean total bilirubin, mg/dl (SD) & $0.742(0.402)$ & $0.942(0.630)$ & 0.354 & $1.31(1.14)$ & $0.83(0.50)$ \\
\hline Mean INR (SD) & $1.12(0.125)$ & $1.14(0.112)$ & 0.629 & $1.11(0.13)$ & $1.15(0.10)$ \\
\hline Child-Pugh Stage (\%) & & & 0.377 & & \\
\hline A & $18(94.7)$ & $22(78.6)$ & & $10(83.3)$ & $12(75)$ \\
\hline $\mathrm{B}$ & $1(5.3)$ & $6(21.4)$ & & $2(16.6)$ & $4(25.0)$ \\
\hline Partial resection prior to treatment $(\%)$ & $1(5.3)$ & $3(10.7)$ & 0.638 & $2(16.6)$ & $1(6.3)$ \\
\hline
\end{tabular}

SIR criteria, one major complication (SIR Grade C) occurred during follow-up: a liver abscess evolved in the ablated area and was treated by CT guided drainage and antibiotics. Additionally, small perihepatic or inguinal hematoma as well as small amounts of free fluid in the pelvis were seen in postinterventional ultrasound in 11 patients (SIR Grade A). Table II provides an overview of the adverse events that occurred in both study arms.

The local recurrence rate was $7.7 \%$ (6/78 lesions), with no difference with regards to the medication used for embolization. There was no statistically significant tendency towards a particular size or location of these recurrent tumors.

Overall survival. Median overall survival was 28.9 months in the TACE group and 32.3 months in the TAE group, with no statistically significant difference between both arms $(p=0.307)$ (Figure 3).

As there were no significant differences in survival between the treatment groups, the survival was analyzed according to BCLC stages in a second step. Patients had a median survival of 32.3 months, 36.9 months and 17.7 months in BCLC stage A, B and C, respectively (Figure 4).
Table II. Overview of adverse events that occurred during the study according to the SIR criteria. No statistically significant difference was observed regarding the occurrence of adverse events in the TACE arm versus the bland embolization arm. The p-Value was determined using the Fisher's Exact Test.

\begin{tabular}{llll}
\hline Complications (SIR Grade) & TACE $(\mathrm{n}=28)$ & TAE $(\mathrm{n}=19)$ & $p$-Value \\
\hline Minor (Grade A) & $7(25 \%)$ & $4(21 \%)$ & 1 \\
Major (Grade C) & $1(3.6 \%)$ & $0(0 \%)$ & 1 \\
Total & $8(28.6 \%)$ & $4(21 \%)$ & 0.74 \\
\hline
\end{tabular}

Time to progression. Following a median follow-up period of 28.3 months, 31 of the 47 patients $(66.0 \%)$ had passed away and 2 patients $(4.2 \%)$ underwent liver transplantation and were excluded from the survival analysis at the timepoint of liver transplantation. Five patients (10.6\%) were lost to follow-up. Median TTP was 10.3 months in the TAE group, and 11.7 months in the TACE group, with no statistically significant difference between the two arms $(p=0.466)$. 

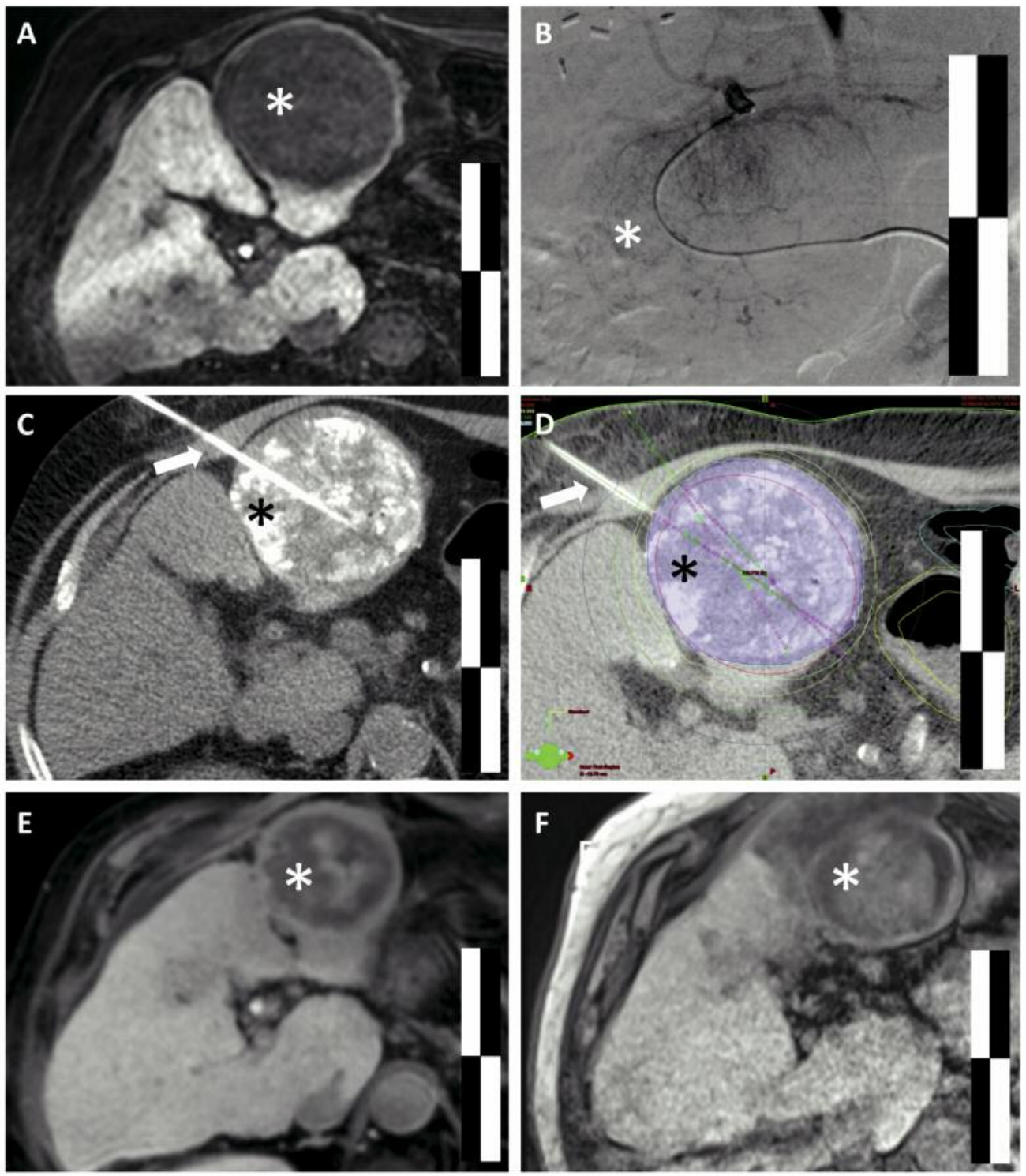

Figure 1. Treatment process of a patient treated with Chemoembolization and CT-HDRBT. (A) Shows the pretreatment MRI imaging of the tumor. (B) Shows the hypervascularisation of the tumor on DSA before Chemoembolization. The good delineation of the tumor on native CT imaging due to the Lipiodol deposition is shown in $(C)$. (D) Depicts the dose planning of the CT-HDRBT following the placement of the catheters. $(E)$ and $(F)$ show the shrinkage of the tumor on follow-up imaging after 9 and 20 months, respectively. *Indicates the tumor while the arrows point to the CTHDRBT catheters. Scale bars: $5 / 10 \mathrm{~cm}$.

\section{Discussion}

The main finding of this study is that the overall survival of patients with HCC treated with the combination therapy showed very promising results compared to the survival data obtained by D' Avola et al. or the EASL, where the expected survival of patients with the allocated therapy in BCLC stage B is 16 months (13) or 24 months (14) versus 36.9 months in our cohort. Survival was even more promising in patients with advanced tumor stage and portal venous infiltration treated with embolization followed by CT-HDRBT, in which the examined median OS was 16.9 months while survival was expected to be only 9 months (13) -10.7 months (14) in BCLC stage $C$.

Interestingly, no radiosensitizing effect of either cisplatin or the alternative drug mitomycin $\mathrm{C}$ was observed in patients treated with TACE and CT-HDRBT, as their survival and local recurrence rates were similar to those of patients treated 

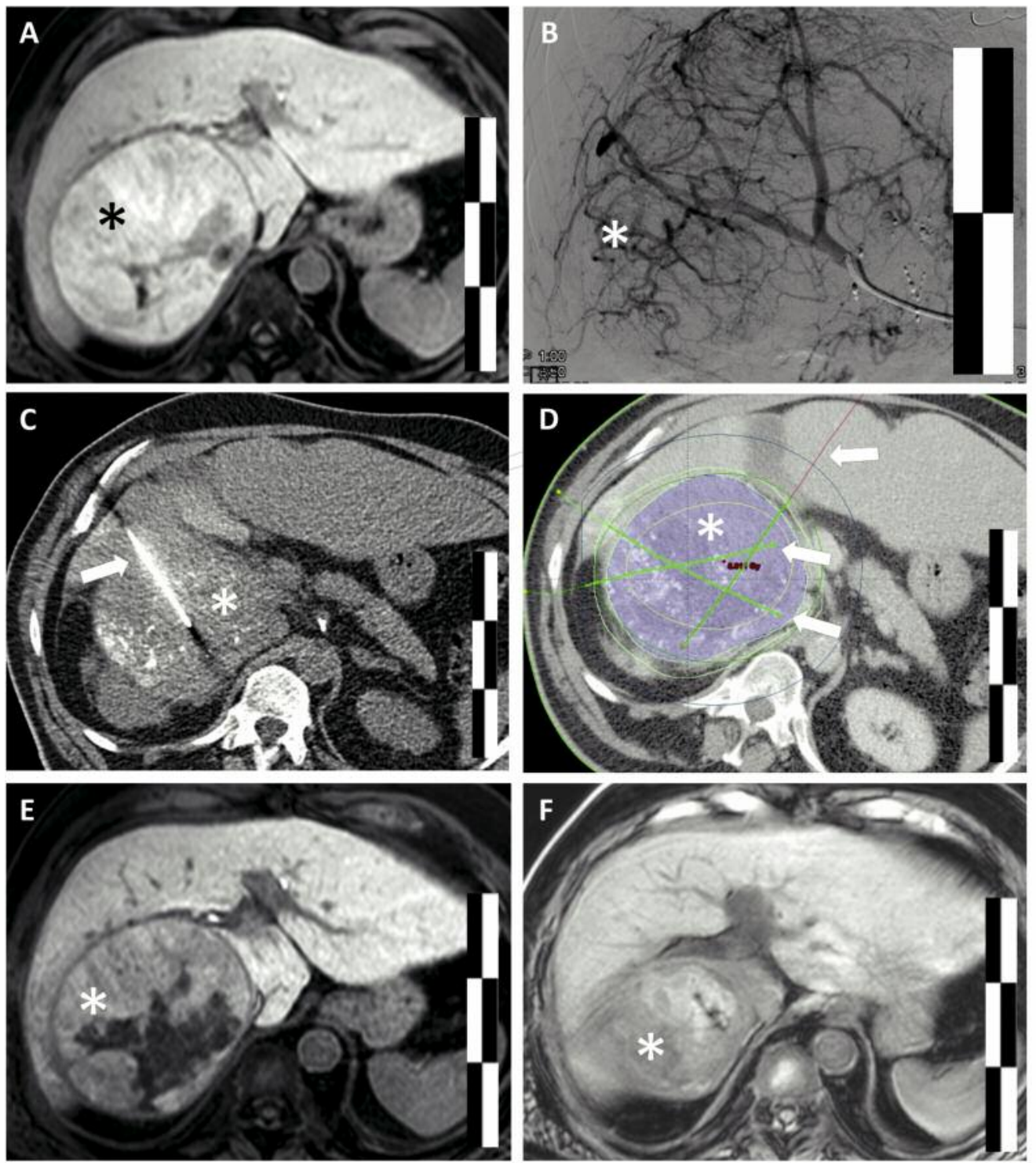

Figure 2. Treatment process of a patient treated with the combination of blande embolization and CT-HDRBT. (A) Shows the pretreatment MRI imaging of the tumor. (B) Shows the hypervascularisation of the tumor on DSA before Chemoembolization. The good delineation of the tumor on native CT imaging due to the Lipiodol deposition is shown in C. (D) Depicts the dose planning of the CT-HDRBT after placement of the catheters. $(E)$ and $(F)$ show the shrinkage and necrosis inside the tumor on follow-up imaging after 3 and 15 months, respectively. ${ }^{*}$ Indicates the tumor while the arrows point to the CT-HDRBT catheters. Scale bars: $5 / 10 \mathrm{~cm}(B)$ and 5/10/15 cm $(A, C-F)$.

with TAE and CT-HDBRT. Our findings are comparable to the data obtained from a meta-analysis by Facciorusso et al., that showed no superiority of TACE over TAE (9).

The local recurrence rate observed in our study was very low $(6.4 \%)$, being indicative of a good local tumor control, despite the large size of the main lesions that reached up to $14 \mathrm{~cm}$. The tumor size investigated in this study is beyond the limits described for other combination therapies, such as radiofrequency ablation following embolization.
CT-HDRBT, as a treatment for hepatic malignancies, was established at our institution in the last two decades and is being used with very promising results in multiple different tumor entities, especially in large size primary hepatic tumors, such as HCCs or cholangiocarcinomas $(8,15)$.

Initial attempts to combine embolization with subsequent ablation were published by Buscarini et al. in 1999 (16). This group concluded that the combination of both these techniques treated HCC tumors more effectively than one 
A

Time To Local Progression

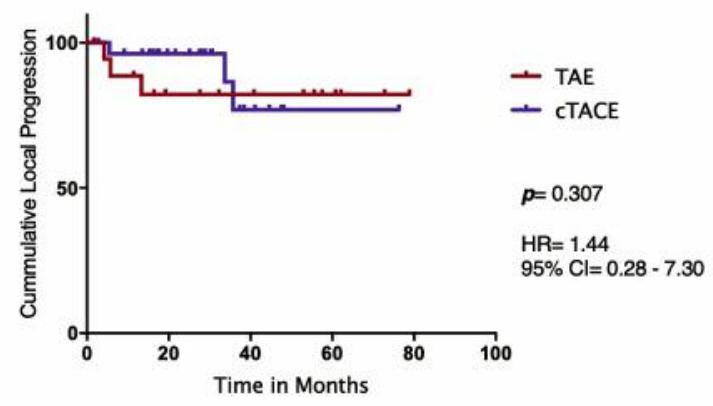

C

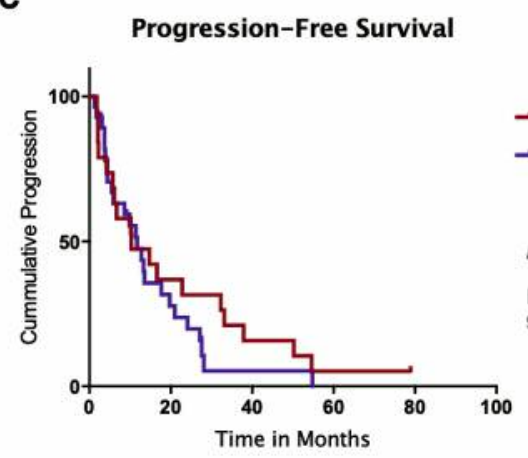

B

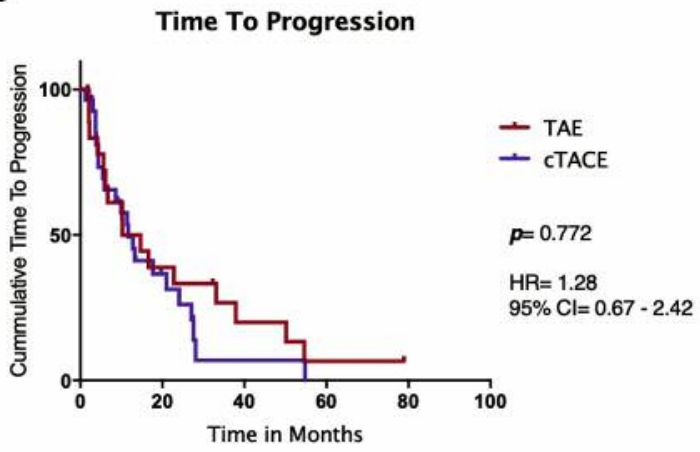

D

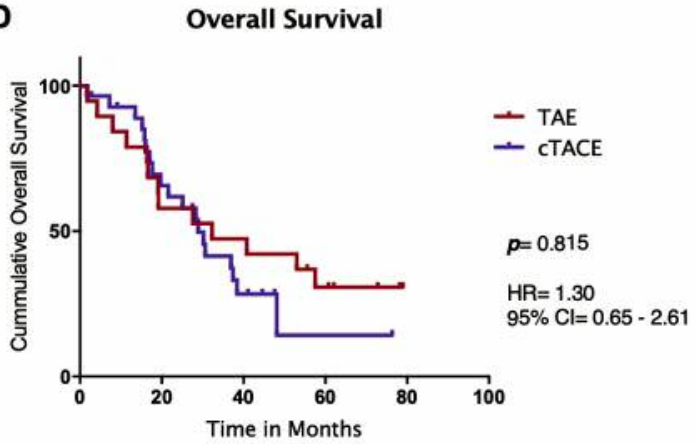

Figure 3. Kaplan-Meier curves of the cohort for time to local progression (A), time to progression (B), progression-free survival (C) and overall survival $(D)$. Red represents the study arm treated with the combination of bland embolization and brachytherapy while blue represents the study arm treated with the combination of chemoembolization and brachytherapy.

type of treatment alone in a cohort of 11 patients. In another study, Liao et al. showed in a series of 36 patients and 41 lesions that tumors of up to $5 \mathrm{~cm}$ can be treated successfully by combination therapy, and pushed the upper size limit further beyond the established boundaries of that time (17). In 2004, Akamatsu et al. conducted a randomized trial with 42 patients, in which it was demonstrated that TAE can be useful before percutaneous ethanol injection in terms of reducing the risk of local tumor recurrence (18). In this study, no patients developed local recurrence after RFA but the mean tumor size was only $29 \pm 11 \mathrm{~mm}$. A possible interventional alternative to treat patients with large size and portal vein infiltrating tumors is radioembolization. The recently presented SIRVENIB trial from the Asian pacific region included 360 patients from 27 centers in 11 Asian countries (19). This trial demonstrated statistically significant longer tumor response rates and fewer severe adverse advents when comparing radioembolization to sorafenib, but no significant improvement in OS was observed. A major drawback of this study was that $28.6 \%$ of the patients in the radioembolization arm did not receive the allocated intervention, whereas the dropout-rate was only $9 \%$ in the

\section{Overall Survival}

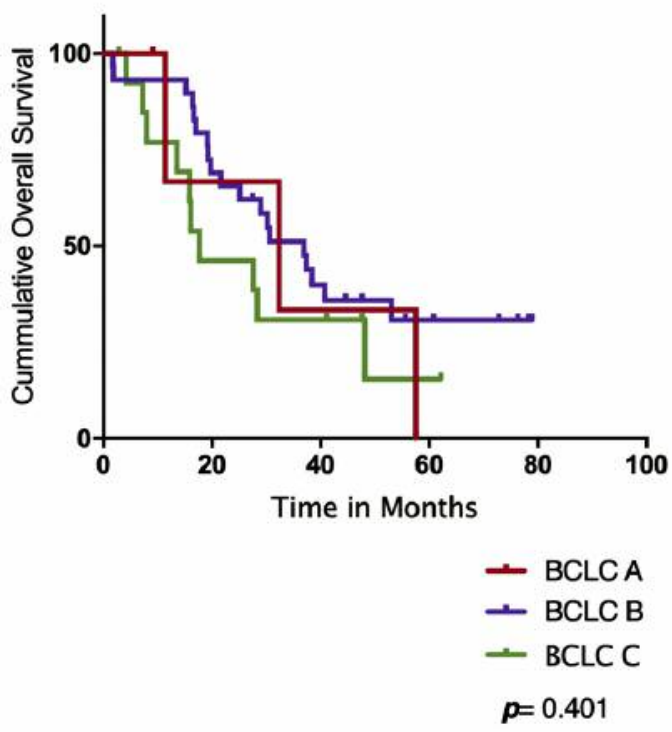

Figure 4. Kaplan-Meier curve for overall survival of the whole cohort grouped based on the BCLC staging system. Red, blue and green represent patients in $B C L C$ stage $A, B$ and $C$, respectively. 
Sorafenib arm. Similar results were observed in the SARAH Trial from France investigating 467 patients, with a median OS of 8.0 months in the radioembolization arm and 9.9 months in the Sorafenib arm (20). In this study, $26.6 \%$ of patients assigned to radioembolization also did not receive the allocated therapy (dropout rate $26.5 \%$ ). In both these trials, the majority of patients had more advanced tumor stages with multifocal disease and a larger extent of portal venous infiltration compared to our cohort. Nevertheless, the low survival rates under both therapies on their own highlight the potential advantage for the survival of advanced stage HCC patients when combination therapy (embolization and CT-HDRBT) is being used.

The major limitation of this study is its retrospective nature and that the therapy allocation had to be deployed at the interventionalists' discretion, since the medical ethics review board denied the randomization of patients for TACE versus bland embolization. The cohort size of 47 patients included in this study is within the typical scope of interventional studies combining two procedures in a single center, however, the results of this study should be confirmed by multi-center studies with a larger cohort. Unfortunately, no group of patients treated with CT-HDRBT only was enrolled in our study. Therefore, the prolonged survival cannot be attributed to a specific part of the treatment. As the institutions' standard approach is to treat all patients with large HCC with the combination of embolization and CT-HDRBT, no other comparable group of patients could be matched to the study cohort retrospectively.

Apart from the oncological outcome, the combination of the embolization and subsequent CT-HDRBT offers two major advantages for the treatment and aftercare of the patient. First, the excellent visibility of the tumor on native CT imaging due to the iodized oil deposition facilitates the optimal catheter placement for radiation. Second, the tumor devascularization occurring following embolization makes it easier to identify new enhancement on follow-up imaging and thus facilitates the detection of progressive disease. Finally, the combination therapy shows no severe postinterventional bleeding.

The combination of transarterial embolization and subsequent high-dose rate brachytherapy shows very promising results with regard to local tumor control and a substantial effect on the survival of patients with large-size HCC. The additional administration of a chemotherapeutic agent during embolization, however, demonstrated no benefit for patients' survival and tumor recurrence over pure embolization.

\section{Conflicts of Interest}

All Authors declare that they have no conflicts of interest.

\section{Authors' Contributions}

Development and Creation of the study was performed by MJP and BG, acquisition of data was done by DS, BRT and MJP. Analysis and interpretation of data was performed by DS, BRT and FC. DS, BRT and BG drafted the manuscript, and participated in the critical revisions together with $\mathrm{FC}$ and $\mathrm{BH}$.

\section{References}

1 Vogel A, Cervantes A, Chau I, Daniele B, Llovet JM, Meyer T, Nault JC, Neumann U, Ricke J, Sangro B, Schirmacher P, Verslype C, Zech CJ, Arnold D and Martinelli E: Hepatocellular carcinoma: Esmo clinical practice guidelines for diagnosis, treatment and follow-up. Ann Oncol 29(4): 238-255, 2018. DOI: 10.1093/annonc/mdy510

2 Llovet JM and Bruix J: Systematic review of randomized trials for unresectable hepatocellular carcinoma: Chemoembolization improves survival. Hepatology 37(2): 429-442, 2003. PMID: 12540794. DOI: 10.1053/jhep.2003.50047

3 Chung-Mau L, Henry N, Wai-Kuen T, Chi-Leung L, Chi-Ming L, Tung-Ping PR, Sheung-Tat F and John W: Randomized controlled trial of transarterial lipiodol chemoembolization for unresectable hepatocellular carcinoma. Hepatology 35(5): 11641171, 2002. PMID: 11981766. DOI: 10.1053/jhep.2002.33156

4 Iezzi R, Pompili M, Posa A, Coppola G, Gasbarrini A and Bonomo L: Combined locoregional treatment of patients with hepatocellular carcinoma: State of the art. World J Gastroenterol 22(6): 19351942, 2016. PMID: 26877601. DOI: 10.3748/wjg. v22.i6.1935

5 Tian G, Yang S, Yuan J, Threapleton D, Zhao Q, Chen F, Cao $\mathrm{H}$, Jiang $\mathrm{T}$ and $\mathrm{Li} \mathrm{L}$ : Comparative efficacy of treatment strategies for hepatocellular carcinoma: Systematic review and network meta-analysis. BMJ Open 8(10): e021269, 2018. PMID: 27082558. DOI: 10.1136/bmjopen-2017-021269

6 Zuo TY, Liu FY, Wang MQ and Chen XX: Transcatheter arterial chemoembolization combined with simultaneous computed tomography-guided radiofrequency ablation for large hepatocellular carcinomas. Chin Med J (Engl) 130(22): 26662673, 2017. PMID: 29133753. DOI: 10.4103/0366-6999.218002

7 Ricke J, Wust P, Wieners G, Beck A, Cho CH, Seidensticker M, Pech M, Werk M, Rosner C, Hänninen EL, Freund T and Felix R: Liver malignancies: Ct-guided interstitial brachytherapy in patients with unfavorable lesions for thermal ablation. J Vasc Interv Radiol 15(11): 1279-1286, 2004. PMID: 15525748. DOI: 10.1097/01.RVI.0000141343.43441.06

8 Collettini F, Schnapauff D, Poellinger A, Denecke T, Schott E, Berg T, Wust P, Hamm B and Gebauer B: Hepatocellular carcinoma: Computed-tomography-guided high-dose-rate brachytherapy (ct-hdrbt) ablation of large $(5-7 \mathrm{~cm})$ and very large (>7 cm) tumours. Eur Radiol 22(5): 1101-1109, 2012. PMID: 22173693. DOI: 10.1007/s00330-011-2352-7

9. Candelaria M, Garcia-Arias A, Cetina L and Duenas-Gonzalez A: Radiosensitizers in cervical cancer. Cisplatin and beyond. Radiat Oncol 1(15), 2006. PMID: 16722549. DOI: 10.1186/1748-717X$1-15$

10. Association WM: World medical association declaration of helsinki: Ethical principles for medical research involving human subjectsworld medical association declaration of helsinkispecial communication. JAMA 310(20): 2191-2194, 2013. PMID: 24141714. DOI: 10.1001/jama.2013.281053 
11. Mohnike K, Wieners G, Schwartz F, Seidensticker M, Pech M, Ruehl R, Wust P, Lopez-Hanninen E, Gademann G, Peters N, Berg T, Malfertheiner P and Ricke J: Computed tomographyguided high-dose-rate brachytherapy in hepatocellular carcinoma: Safety, efficacy, and effect on survival. Int J Radiat Oncol Biol Phys 78(1): 172-179, 2010. PMID: 20056348. DOI: 10.1016/j.ijrobp.2003.07.1700

12 Goldberg SN, Grassi CJ, Cardella JF, Charboneau JW, Dodd GD, 3rd, Dupuy DE, Gervais D, Gillams AR, Kane RA, Lee FT Jr., Livraghi T, McGahan J, Phillips DA, Rhim H and Silverman SG: Image-guided tumor ablation: Standardization of terminology and reporting criteria. J Vasc Interv Radiol 20(7): S377-390, 2009. PMID: 19560026. DOI: 10.1016/j.jvir.2009.04.011

13 Easl clinical practice guidelines: Management of hepatocellular carcinoma. J Hepatol 69(1): 182-236, 2018. PMID: 29628281. DOI: 10.1016/j.hep.2018.03.019

14 D'Avola D, Inarrairaegui M, Pardo F, Rotellar F, Marti P, Bilbao JI, Martinez-Cuesta A, Benito A, Alegre F, Mauleon E, Herrero JI, Quiroga J, Prieto J and Sangro B: Prognosis of hepatocellular carcinoma in relation to treatment across bclc stages. Ann Surg Oncol 18(7): 1964-1971, 2011. PMID: 21267791. DOI: 10.1245/ s10434-011-1551-4

15 Schnapauff D, Denecke T, Grieser C, Collettini F, Seehofer D, Sinn M, Banzer J, Lopez-Hanninen E, Hamm B, Wust P and Gebauer B: Computed tomography-guided interstitial hdr brachytherapy (ct-hdrbt) of the liver in patients with irresectable intrahepatic cholangiocarcinoma. Cardiovasc Intervent Radiol 35(3): 581-587, 2012. PMID: 21833806. DOI: 10.1007/s00270011-0249-0

16 Buscarini L, Buscarini E, Di Stasi M, Quaretti P and Zangrandi A: Percutaneous radiofrequency thermal ablation combined with transcatheter arterial embolization in the treatment of large hepatocellular carcinoma. Ultraschall Med 20(2): 47-53, 1999. PMID: 10407974. DOI: 10.1055/s-1999-14233

17 Liao GS, Yu CY, Shih ML, Chan DC, Liu YC, Yu JC, Chen TW and Hsieh CB: Radiofrequency ablation after transarterial embolization as therapy for patients with unresectable hepatocellular carcinoma. Eur J Surg Oncol 34(1): 61-66, 2008. PMID: 17434711. DOI: 10.1016/j.ejso.2007.02.006
18 Masatoshi A, Haruhiko Y, Shuntaro O, Shinpei S, Yukihiro K, Tomonori F, Ryosuke T, Masatoshi I, Keisuke H, Takuma T, Shuichiro S, Takashi I and Masao O: Evaluation of transcatheter arterial embolization prior to percutaneous tumor ablation in patients with hepatocellular carcinoma: A randomized controlled trial. Liver Int 24(6): 625-629, 2004. PMID: 15566514. DOI: 10.1111/j.1478-3231.2004.0963.x

19 Chow PKH, Gandhi M, Tan S-B, Khin MW, Khasbazar A, Ong J, Choo SP, Cheow PC, Chotipanich C, Lim K, Lesmana LA, Manuaba TW, Yoong BK, Raj A, Law CS, Cua IHY, Lobo RR, Teh CSC, Kim YH, Jong YW, Han H-S, Bae S-H, Yoon H-K, Lee R-C, Hung C-F, Peng C-Y, Liang P-C, Bartlett A, Kok KYY, Thng C-H, Low AS-C, Goh ASW, Tay KH, Lo RHG, Goh BKP, Ng DCE, Lekurwale G, Liew WM, Gebski V, Mak KSW, Soo KC and Group oboA-PHCT: Sirvenib: Selective internal radiation therapy versus sorafenib in asia-pacific patients with hepatocellular carcinoma. J Clin Oncol 36(19): 1913-1921, 2018. PMID: 29498924. DOI: 10.1200/jco.2017.76.0892

20 Vilgrain V, Pereira H, Assenat E, Guiu B, Ilonca AD, Pageaux G-P, Sibert A, Bouattour M, Lebtahi R, Allaham W, Barraud H, Laurent V, Mathias E, Bronowicki J-P, Tasu J-P, Perdrisot R, Silvain C, Gerolami R, Mundler O, Seitz J-F, Vidal V, Aubé C, Oberti F, Couturier O, Brenot-Rossi I, Raoul J-L, Sarran A, Costentin C, Itti E, Luciani A, Adam R, Lewin M, Samuel D, Ronot M, Dinut A, Castera L and Chatellier G: Efficacy and safety of selective internal radiotherapy with yttrium-90 resin microspheres compared with sorafenib in locally advanced and inoperable hepatocellular carcinoma (sarah): An open-label randomised controlled phase 3 trial. Lancet Oncol 18(12): 16241636, 2017. PMID: 29107679. DOI: 10.1016/S1470-2045(17) 30683-6 